\title{
Identification of a novel heterozygous SPTB mutation by whole genome sequencing in a Chinese patient with hereditary spherocytosis and atrial septal defect: a case report
}

\author{
Zhanhui Du, Gang Luo, Kuiliang Wang, Zhen Bing and Silin Pan*
}

\begin{abstract}
Background: Hereditary spherocytosis (HS) is a common inherited red blood cell membrane disorder characterized by an abnormal increase of spherocytes in peripheral blood. SPTB gene mutation is one of the most common causes of HS; however, few cases of HS resulting from SPTB mutation in the Chinese population have been reported so far.

Case presentation: A 3-year-old Chinese girl presented to Qingdao Women and Children's Hospital, Qingdao University, with atrial septal defect (ASD). Meanwhile, she was clinically diagnosed with HS. Whole genome sequencing (WGS) was performed for the proband and her parents for genetic molecular analysis. A novel SPTB mutation (c.1756delG) was detected by WGS and confirmed by Sanger sequencing in the proband. This mutation results in a frameshift with a premature termination codon in exon 12, leading to a nonsense mutation (p.Ala586Profs*7). Her parents had no similar symptoms, and blood routine and serum biochemical tests showed no significant abnormalities. The patient's mother did not know of any relatives with HS-like symptoms. Percutaneous transcatheter closure was successfully performed for treating the ASD.

Conclusion: In this study, we identified a novel SPTB frameshift mutation in a Chinese girl with HS. This finding would expand the spectrum of SPTB mutations, provide a valuable insight into the genotyping of HS in the Chinese population, and contribute to the clinical management and genetic counseling in HS.
\end{abstract}

Keywords: Hereditary spherocytosis, Atrial septal defect, SPTB mutation, Whole genome sequencing, Case report

\section{Background}

Hereditary spherocytosis (HS) is a common inherited red blood cell (RBC) membrane disorder characterized by an abnormal increase of spherocytes in peripheral blood. HS occurs in all racial and ethnic groups, and its prevalence in China is about $1.27-1.49$ per 100,000

* Correspondence: silinpan@126.com

Heart center, Qingdao Women and Children's Hospital, Qingdao University, 217 Liaoyangxi Road, Qingdao 266034, China individuals [1]. The HS clinical spectrum ranges broadly from a nearly asymptomatic disease to life-threatening anemia, serious splenomegaly, and/or severe hyperbilirubinemia, even in the same family. About $70 \%$ of HS cases are associated with autosomal dominant inheritance, although non-dominant and autosomal recessive modes of inheritance have been described.

HS is mostly characterized by sphere-shaped erythrocytes on peripheral blood smear resulting from

C C The Author(s). 2021 Open Access This article is licensed under a Creative Commons Attribution 4.0 International License, which permits use, sharing, adaptation, distribution and reproduction in any medium or format, as long as you give appropriate credit to the original author(s) and the source, provide a link to the Creative Commons licence, and indicate if changes were made. The images or other third party material in this article are included in the article's Creative Commons licence, unless indicated otherwise in a credit line to the material. If material is not included in the article's Creative Commons licence and your intended use is not permitted by statutory regulation or exceeds the permitted use, you will need to obtain permission directly from the copyright holder. To view a copy of this licence, visit http://creativecommons.org/licenses/by/4.0/ The Creative Commons Public Domain Dedication waiver (http://creativecommons.org/publicdomain/zero/1.0/) applies to the data made available in this article, unless otherwise stated in a credit line to the data. 
qualitative and/or quantitative abnormalities of $\mathrm{RBC}$ membrane proteins, which are basically caused by mutations in the corresponding genes. Current evidence indicates that mutations in the ankyrin 1 (ANK1; about $50 \%$ ), spectrin- $\beta$, erythrocytic (SPTB; approximately $20 \%$ ), solute carrier family 4, member 1 (SLC4A1; about $15 \%)$, erythrocyte membrane protein band 4.2 (EPB42; $10 \%)$ and spectrin-a, erythrocytic 1 (SPTA1; 5\%) are associated with membrane defects in HS [2].

Although SPTB mutations mostly cause HS, only few HS families with such mutations have been reported in the Chinese population [3]. Here, we identified a novel SPTB frameshift mutation responsible for $\mathrm{HS}$ in a Chinese family by whole genome sequencing (WGS).

\section{Case presentation}

\section{Clinical phenotype}

A 3-year-old Chinese girl was referred to Qingdao Women and Children's Hospital, Qingdao University on April 20, 2017, after a diagnosis of atrial septal defect (ASD) and HS. ASD in this patient was incidentally detected during a physical examination 2 years ago in a local hospital. Subsequent echocardiographic data confirmed this diagnosis, and the girl was suggested to undergo regular check-up. The patient had experienced bouts of weakness and easy fatigability since birth. One month ago, she visited a local hospital for anemia. Based on laboratory findings and the osmotic fragility test, a diagnosis of HS was made. Our hospital was recommended for further treatment.

On admission, the patient's sclerae and skin were icteric, and mucosal membranes were pale. Physical examination revealed a systolic ejection murmur with splitting of the second heart sound at the left second intercostal space, and an enlarged spleen in the left subcostal region. Hematologic assessment revealed hemoglobin levels at $8.2 \mathrm{~g} / \mathrm{dl}$ (normal range, $11-17 \mathrm{~g} / \mathrm{dl}$ ), hematocrit at $23.8 \%$ (normal range, $36-56 \%$ ), RBC count at $3.15 \times 10^{12} / \mathrm{L}$ (normal range, $3.50-5.30 \times 10^{12} / \mathrm{L}$ ), and white blood cell count at $6.52 \times 10^{9} / \mathrm{L}$ (normal range, $\left.4.0-10.0 \times 10^{9} / \mathrm{L}\right)$. Total serum bilirubin $(89.4 \mu \mathrm{M}$; normal range, $2.0-22.0 \mu \mathrm{M})$, direct bilirubin $(11.8 \mu \mathrm{M}$; normal range, $0.0-8.0 \mu \mathrm{M})$, and indirect bilirubin $(77.6 \mu \mathrm{M}$; normal range, $0.0-14.0 \mu \mathrm{M}$ ) levels were significantly increased. Liver enzyme levels were normal. RBC size showed disparity, and the presence of spherocytes (about $12 \%$ ) was noted on peripheral blood smear (Fig. 1A). Bone marrow smear analysis showed active proliferation with erythroid preponderance (myeloid cells was 39.5\%, and erythroid cell was $48.5 \%$ ), increase of intermediate and late erythroblast, different size of mature erythrocyte, and the presence of polychromatic and spherical erythrocytes (Fig. 1B). RBCs showed elevated osmotic fragility. Echocardiography revealed a fossa ovalis ASD with left-to-right shunt, enlarged right atrium and right ventricle, and dilated main pulmonary artery.

The girl was the only child born to healthy nonconsanguineous parents (Fig. 1C). Because of "too little amniotic fluid" she was born at 37 weeks of gestation by cesarean section, with a birth weight of $3000 \mathrm{~g}$. It is unclear whether the mother had any specific medication or exposure history during pregnancy. Her parents had no similar symptoms, and their blood routine and serum biochemical tests showed no significant abnormalities. The patient's mother did not know of any relatives with HS-like symptoms.

The conventional treatment option for ASD is open heart surgery with cardiopulmonary bypass, which would put the HS patient at risk of hemolytic anemia because of the deleterious effects of the heart-lung machine. Hence, percutaneous transcatheter closure for ASD was successfully performed. Because the patient lived in another province, there was no follow-up after discharge on May 7, 2017.

\section{Molecular analysis}

Genetic molecular analysis was performed for the proband and her parents to assess the genetic cause of HS. The study protocol was approved by the Ethics Committee of Qingdao Women and Children's Hospital, Qingdao University. Written informed consent for clinical and molecular analyses was obtained from all subjects.

WGS was performed by BGI (Shenzhen, China) following the standard procedure. Sequencing data were filtered by removing low-quality reads, adapters, and sequences with more than $5 \%$ unknown bases. Clean reads were matched to human reference genome 19 (hg19) using the Burrows-Wheeler Alignment tool. Duplications were marked with Picard (http:// broadinstitute.github.io/picard) and BAM was sorted with Samtools2. GATK Best Practices Pipeline, which includes Local realignment around indels, Base quality score recalibration (BQSR), Haplotype Caller, and Variant Quality Score Recalibration (VQSR), was used in the process of SNP and INDEL calling. After filtering highquality variants, SNPs and INDELs were annotated using the snpEff 3, dbSNP (version 147), 1000 genomes (August 2015), ExAC (version 3), and dbNSFP (version 2.9) databases.

A total of 20,154 sequence variants were identified and subjected to interpretation, according to the variant interpretation guidelines of the American College of Medical Genetics and Genomics (ACMG) [4]. All the identified variants were firstly filtered using the dbSNP, HapMap, 1000 project, and 100 Chinese healthy adults' local databases, deleting all variants with frequency $>1 \%$ in the healthy population. Then, all the remaining 


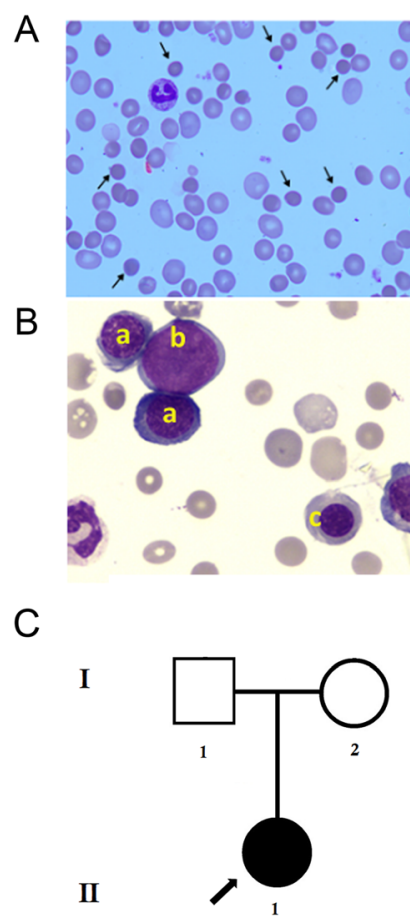

D Patient



Father

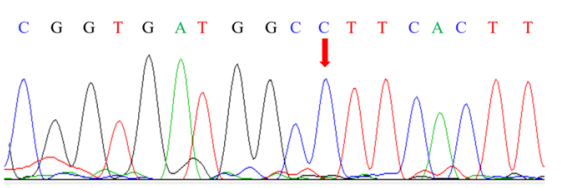

Mother

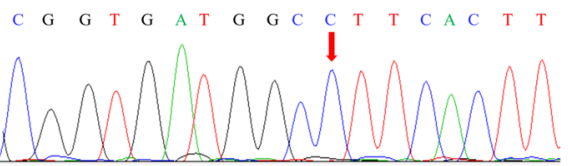

$\mathrm{E}$

1681 TTGGAGGTTGAAAGACCTGCTACAGAAGCACAAGTTGATGGAAGCTGACATCGCCATCCAA $\begin{array}{llllllllllllllllllllll}561 & \text { L } & \text { E } & \text { V } & \text { E } & \text { D } & \text { L } & \text { L } & \text { Q } & \text { K } & \text { H } & \text { K } & \text { L } & \text { M } & \text { E } & \text { A } & \text { D } & \text { I } & \text { A } & \text { I } & Q\end{array}$

1741 GGGGACAAAGTGAAGGCCATCACCGCAGCCACCCTGAAGTTCACCGAGGGGAAAGGGTAC

1681 TTGGAGGTTGAAGACCTGCTACAGAAGCACAAGTTGATGGAAGCTGACATCGCCATCCAA

$\begin{array}{lllllllllllllllllllllll}561 & \text { L } & \text { E } & \text { V } & \text { E } & \text { D } & \text { L } & \text { I } & Q & \text { K } & \text { K } & \text { H } & \text { K } & \text { I } & \text { M } & \text { E } & \text { A } & \text { D } & \text { I } & \text { A } & \text { I } & Q\end{array}$

1741 GGGGACAAAGTGAAGCCATCACCGCAGCCACCCTG
581 G

Fig. 1 Identification of a novel SPTB frameshift mutation. A. Peripheral blood smear of the proband showing moderate spherocytosis (Wright'sGiemsa staining) (abnormal cells indicated by arrows). B. Wright's-Giemsa staining of the proband's bone marrow smear demonstrating erythroblastic hyperplasia dominated with rubricytes and metarubricytes: a, intermediate erythroblasts; b, myeloblasts; C, late erythroblasts. C. Pedigree of the family with a hereditary spherocytosis (HS) case. D. Sanger sequencing confirming the heterozygous mutation of SPTB (c.1756delG). E. The deletion mutation leads to the formation of a truncated SPTB protein (p.Ala586Profs*7)

filtered variants were searched in the OMIM and CGD databases for identifying the variants of genes associated with the disease phenotype. Then, the selected variants were further searched in published literatures in Google scholar, PubMed and the Human Gene Mutation Database (HGMD) [5]. The identified variants were also predicted by in silico software such as SIFT, Polyphen-2, Mutation Taster, and PROVEAN. The classification of the newly identified variants was based on the ACMG Guidelines [4].

Sanger sequencing was performed for validating the variants identified by WGS. Primers were designed based on reference human genome sequences in NCBI-GenBank. Amplification was performed on an ABI 9700 Thermal Cycler (Applied Biosystems, Foster City, CA, USA). The amplified products were subjected to direct sequencing on an ABI PRISM 3730 automated sequencer (Applied Biosystems). DNAS TAR SeqMan (DNASTAR, Madison, Wisconsin, USA) was used for comparing and analyzing the sequencing data. The primer sequences were: F, 5' ${ }^{\prime}$ TCCAAG TTGGGTTGTTAGGC-3'; R, 5'-GTTCTCTTTG CCAGGCTCAC-3'.
As a result, a heterozygous variant c.1756delG was identified in exon 12 of the SPTB gene in the proband and confirmed as a de novo variant because Sanger sequencing of her parents showed a wild type (Fig. 1D). The pedigree of the proband was shown in Fig. 1C. This mutation causes a frameshift by the formation of a premature stop codon. This frameshift mutation leads to the synthesis of a truncated SPTB protein (p.Ala586Profs*7) comprising 593 amino acids instead of 2137 amino acids in the wild type counterpart (Fig. 1E). The SPTB variant is predicted as deleterious, probably damaging, disease causing and deleterious by SIFT, Polyphen-2, MutationTaster, and PROVEAN and a high frequency of de novo mutations being reported in the SPTB gene (Table 1). Hence, it is a loss-of-function mutation. Sanger sequencing revealed that this mutation was novel, which was not present in the proband's unaffected parents, 100 other healthy individuals, ExAC, HGMD or 1000 Genome databases. Hence, a novel de novo SPTB mutation c.1756delG was confirmed in the Chinese girl. All data used for the analysis in this study are available in the CNGB Nucleotide Sequence Archive 
Table 1 Previously reported hereditary spherocytosis cases with SPTB mutations

\begin{tabular}{|c|c|c|c|c|c|c|}
\hline Author \& year & Number of Patients & Exon & Nucleotide & Amino acid & Type & Mutation type \\
\hline \multirow[t]{11}{*}{ Park 2016 [6] } & 1 & 5 & c.624_640delinsACCTCC & p.Phe208Leufs*12 & Heterogeneous & Frameshift \\
\hline & 2 & 11 & $\begin{array}{l}\text { c.1493_1502delGCAT } \\
\text { CACGGC }\end{array}$ & p.Arg498Profs*72 & Heterogeneous & Frameshift \\
\hline & 1 & 12 & c.1795 + 2_1795 + 3delTG & & Heterogeneous & Splicing error \\
\hline & 1 & 13 & c.2572_2573delGA & p.Asp858Argfs*3 & Heterogeneous & Frameshift \\
\hline & 1 & 14 & $c .2686 G>T$ & p.Glu896* & Heterogeneous & Nonsense \\
\hline & 1 & 15 & c.3058C > A & p.Gln1020Lys & Heterogeneous & Missense \\
\hline & 1 & 15 & c.3205delG & p.Asp1069llefs*59 & Heterogeneous & Frameshift \\
\hline & 1 & 15 & c.3440delA & p.Asp1147Valfs*79 & Heterogeneous & Frameshift \\
\hline & 1 & 18 & c.3976G > T & p.Glu1326* & Heterogeneous & Nonsense \\
\hline & 1 & 20 & c. $4291 C>T$ & p.Arg1431* & Heterogeneous & Nonsense \\
\hline & 1 & 25 & c. $5266 C>\mathrm{T}$ & p.Arg1756* & Heterogeneous & Nonsense \\
\hline \multirow[t]{3}{*}{ Xue 2020 [7] } & 1 & 19 & $c .4181 G>A$ & p.W1394X & Heterogeneous & Nonsense mutation \\
\hline & 1 & 2 & $c .211 G>A$ & p.V71M & Heterogeneous & Missense mutation \\
\hline & 2 & 23 & $c .4973+5 G>A$ & Splicing & Heterogeneous & Splice mutation \\
\hline Meglic 2020 [8] & 7 & Unspecified & c. $4796 \mathrm{G}>\mathrm{A}$ & p.Trp1599Ter & Heterogeneous & Nonsense mutation \\
\hline \multirow[t]{3}{*}{ Xue 2019 [9] } & 2 & 23 & $c .4973+5 G>A$ & splicing & Heterogeneous & Splice mutation \\
\hline & 1 & 19 & $c .4181 G>A$ & p.W1394X & Heterogeneous & Nonsense mutation \\
\hline & 1 & 2 & $c .211 G>A$ & p.V71M & Heterogeneous & Missense mutation \\
\hline van Vuren 2019 [10] & 17 & Unspecified & Unspecified & Unspecified & Unspecified & Unspecified \\
\hline Li 2019 [11] & 1 & Unspecified & c. 2413 C > T & p.Gln805* & Heterogeneous & Nonsense mutation \\
\hline Shen 2019 [12] & 1 & 23 & c. 4873 C > T & p.R1625X & Heterogeneous & Nonsense mutation \\
\hline Choi 2019 [13] & 28 & Unspecified & Unspecified & Unspecified & Unspecified & Unspecified \\
\hline Shin 2018 [14] & 1 & 13 & c. $1956 G>A$ & p.Trp652* & Heterogeneous & Nonsense mutation \\
\hline
\end{tabular}

with accession number CNP000089 (CNSA: https:// db.cngb.org/cnsa).

\section{Discussion and conclusions}

This study described a Chinese family with a member affected by HS. A novel SPTB mutation (c.1756delG) was detected in the proband by WGS, and confirmed by Sanger sequencing. This mutation results in a frameshift with a premature termination codon within exon 12, leading to a nonsense mutation (p.Ala586Profs*7).

The SPTB gene is located on chromosome 14q23.3, and encodes the $\beta$-spectrin protein, which is typically composed of 4 structural domains, including the activity binding domain, dimerization domain, spectrin repeats, and ankyrin binding domain. $\beta$-spectrin forms the cytoskeleton along with a-spectrin, and tethers it to the RBC membrane by interacting with ankyrin to maintain erythrocyte deformability and stability. SPTB mutation is the second most common pathologic mutation in HS, only after ANK1 mutation [2]. A recent trial reported 6 frameshift, 5 nonsense and 1 splicing error mutations in the SPTB gene in patients with HS [6]. Another study assessing 35 Chinese patients with suspected HS reported 3 cases with mutated SLC4A1, 16 with ANK1 mutations, and 16 with mutated $S P T B$, indicating that SPTB and ANK1 are the most commonly mutated genes in Chinese HS patients [15]. In our study, DNA analysis of the patient revealed a $\mathrm{G}$ deletion at the position 1756 that resulted in a frameshift mRNA by premature termination codon within exon 12. This premature termination codon-bearing transcript might be degraded by nonsense-mediated mRNA decay [7] or produce a Cterminal truncated protein, either of which might lead to a $\beta$-spectrin deficiency. Deficient SPTB protein levels due to frameshift mutation in the spectrin repeats domain are considered a cause of HS. The current patient showed increased osmotic fragility, which is a wellknown pathological feature of type 2 HS caused by $S P T B$ mutation. Previously reported hereditary spherocytosis cases with SPTB mutations were summarized in Table $1[8-14,16]$.

Similar to patients with other hemolytic anemia types, HS cases are subject to various problems such as severe splenomegaly with or without gallbladder disease. In this report, the proband showed hepatosplenomegaly. Splenectomy is a very effective treatment for reducing 
hemolysis, leading to significantly prolonged red cell lifespan. However, it should be performed only after careful risk-benefit assessment. The current patient was 3 years old, and it is not recommended to perform splenectomy before cardiac surgery since the immune function should be maintained. Additionally, the patient was diagnosed with ASD. Patients with concurrent ASD and HS are rare. To the best of our knowledge, only four such cases have been reported [17-20]. Percutaneous transcatheter closure for ASD was performed to avoid potential risk of hemolytic anemia.

HS diagnosis is made on clinical suspicion often including a family history and confirmatory blood test. However, about $25 \%$ of patients have a negative family history, which will increase the difficulty of HS diagnosis [21]. Confirming hereditary RBC membrane disorders at the molecular level using next-generation sequencing is important for HS diagnosis, clinical management as well as genetic counseling. WGS enables the screening of all coding regions and deep intronic variants usually causing disease. In this study, a novel $S P T B$ mutation responsible for HS was successfully detected using WGS in a Chinese patient. These results expand the spectrum of SPTB mutations, providing novel insights into the molecular mechanisms involved in HS and confirming WGS as an effective method for identifying novel pathogenic mutations.

\section{Abbreviations}

HS: Hereditary spherocytosis; ASD: Atrial septal defect; WGS: Whole genome sequencing; RBC: Red blood cell; ANK1: Ankyrin 1; SPTB: Spectrin- $\beta$; BQSR: Base quality score recalibration; VQSR: Variant Quality Score Recalibration; ACMG: American College of Medical Genetics and Genomics; HGMD: Human Gene Mutation Database

\section{Acknowledgements}

Not applicable.

\section{Authors' contributions}

ZHD and GL carried out the studies, participated in collecting data, and drafted the manuscript. SLP performed the statistical analysis and participated in its design. KLW and ZB helped to draft the manuscript. All authors read and approved the final manuscript.

\section{Funding}

This work was supported by grants from National Natural Science Foundation of China (81770316, 81970249), the Taishan Scholars Program of Shandong Province (2018), the Medical Science and Technology Development Plan of Shandong Province (2018WS370), and Qingdao Science and Technology Plan (20-3-4-47-nsh). The funders had no role in study design, data collection and analysis, decision to publish, or preparation of the manuscript.

\section{Availability of data and materials}

The datasets used and/or analyzed during the current study are available from the corresponding author on reasonable request.

\section{Declarations}

\section{Ethics approval and consent to participate}

The study protocol was approved by the Ethics Committee of Qingdao Women and Children's Hospital, Qingdao University. Written informed consent for clinical and molecular analyses as well as data publication was obtained from all subjects.

\section{Consent for publication}

Written informed consent to publication in this study was obtained from the participants' legal guardian.

\section{Competing interests}

The authors declare that they have no competing interests.

Received: 7 September 2020 Accepted: 14 June 2021

Published online: 28 June 2021

\section{References}

1. Wang C, Cui Y, Li Y, Liu X, Han J. A systematic review of hereditary spherocytosis reported in Chinese biomedical journals from 1978 to 2013 and estimation of the prevalence of the disease using a disease model. Intractable Rare Dis Res. 2015;4:76-81.

2. Wang X, Yi B, Mu K, Shen N, Zhu Y, Hu Q, et al. Identification of a novel de novo ANK1 R1426* nonsense mutation in a Chinese family with hereditary spherocytosis by NGS. Oncotarget. 2017;8:96791-7.

3. Meng LL, Yuan SM, Tu CF, Lin G, Lu GX, Tan YQ. Next-generation sequencing identified a novel SPTB frameshift insertion causing hereditary spherocytosis in China. Ann Hematol. 2019;98:223-6.

4. Richards S, Aziz N, Bale S, Bick D, Das S, Gastier-Foster J, et al. Standards and guidelines for the interpretation of sequence variants: a joint consensus recommendation of the American College of Medical Genetics and Genomics and the Association for Molecular Pathology. Genet Med. 2015; 17:405-24.

5. Stenson PD, Mort M, Ball EV, Evans K, Hayden M, Heywood S, et al. The human gene mutation database: towards a comprehensive repository of inherited mutation data for medical research, genetic diagnosis and nextgeneration sequencing studies. Hum Genet. 2017;136:665-77.

6. Park J, Jeong DC, Yoo J, Jang W, Chae H, Kim J, et al. Mutational characteristics of ANK1 and SPTB genes in hereditary spherocytosis. Clin Genet. 2016;90:69-78.

7. Silva AL, Romão L. The mammalian nonsense-mediated mRNA decay pathway: to decay or not to decay! Which players make the decision? FEBS Lett. 2009;583:499-505.

8. Xue J, He Q, Xie XJ, Su AL, Cao SB. A clinical and experimental study of adult hereditary spherocytosis in the Chinese population. Kaohsiung J Med Sci. 2020;36:552-60

9. Meglic A, Debeljak M, Kovac J, Trampus Bakija A, Rajic V, Kojc N, et al. SPTB related spherocytosis in a three-generation family presenting with kidney failure in adulthood due to co-occurrence of UMOD disease causing variant. Nefrologia. 2020;40:421-8.

10. Xue J, He Q, Xie X, Su A, Cao S. Clinical utility of targeted gene enrichment and sequencing technique in the diagnosis of adult hereditary spherocytosis. Ann Transl Med. 2019;7:527.

11. van Vuren A, van der Zwaag B, Huisjes R, Lak N, Bierings M, Gerritsen E, et al. The complexity of genotype-phenotype correlations in hereditary spherocytosis: a cohort of 95 patients: genotype-phenotype correlation in hereditary spherocytosis. Hemasphere. 2019;3:e276.

12. Li Y, Li Y, Yang Y, Yang WR, Li JP, Peng GX, et al. Next generation sequencing reveals co-existence of hereditary spherocytosis and DubinJohnson syndrome in a Chinese gril: a case report. World J Clin Cases. 2019; 7:3303-9.

13. Shen $H$, Huang $H$, Luo K, Yi Y, Shi X. Two different pathogenic gene mutations coexisted in the same hereditary spherocytosis family manifested with heterogeneous phenotypes. BMC Med Genet. 2019;20:90.

14. Choi HS, Choi Q, Kim JA, Im KO, Park SN, Park Y, et al. Molecular diagnosis of hereditary spherocytosis by multi-gene target sequencing in Korea: matching with osmotic fragility test and presence of spherocyte. Orphanet J Rare Dis. 2019;14:114.

15. Bogusławska DM, Heger E, Machnicka B, Skulski M, Kuliczkowski K, Sikorski AF. A new frameshift mutation of the $\beta$-spectrin gene associated with hereditary spherocytosis. Ann Hematol. 2017;96:163-5.

16. Shin S, Jang W, Kim M, Kim Y, Park SY, Park J, et al. Targeted nextgeneration sequencing identifies a novel nonsense mutation in SPTB for hereditary spherocytosis: a case report of a Korean family. Medicine (Baltimore). 2018;97:e9677. 
17. Ji Z, Liu N, Du Z, Luo G, Bing Z, Xing Q, et al. Transcatheter closing atrial Septal defect in a child with hereditary spherocytosis. Front Pediatr. 2019;7: 506.

18. Aoyagi S, Kawano H, Tomoeda H, Hiratsuka R, Kawara T. Open heart operation in a patient with hereditary spherocytosis: a case report. Ann Thorac Cardiovasc Surg. 2001;7:375-7.

19. Dal A, Kumar RS. Open heart surgery in presence of hereditary spherocytosis. J Cardiovasc Surg. 1995;36:447-8.

20. Agarwal SK, Binbrek AS, Thompson JA, Siddiqui SA. Massive pulmonary embolism and acute limb ischaemia in a patient of hereditary spherocytosis and patent foramen ovale. Heart Lung Circ. 2010;19:742-4.

21. Perrotta S, Gallagher PG, Mohandas N. Hereditary spherocytosis. Lancet. 2008;372:1411-26

\section{Publisher's Note}

Springer Nature remains neutral with regard to jurisdictional claims in published maps and institutional affiliations.

Ready to submit your research? Choose BMC and benefit from:

- fast, convenient online submission

- thorough peer review by experienced researchers in your field

- rapid publication on acceptance

- support for research data, including large and complex data types

- gold Open Access which fosters wider collaboration and increased citations

- maximum visibility for your research: over $100 \mathrm{M}$ website views per year

At BMC, research is always in progress.

Learn more biomedcentral.com/submissions 